\title{
summary
}

\section{Arthrocentesis with sodium hyaluronate is more effective than arthrocentesis alone in temporomandibular joint treatment}

\begin{abstract}
Alpaslan GH, Alpaslan G. Efficacy of temporomandibular joint arthrocentesis with and without injection of sodium
\end{abstract} hyaluronate in treatment of internal derangements. J Oral Maxillofacial Surg 2001; 59:613-618

Question: Is arthrocentesis with sodium hyaluronate more effective than arthrocentesis alone in people with temporomandibular joint derangements?

Objective To investigate the efficacy of arthrocentesis with and without injection of sodium hyaluronate $(\mathrm{SH})$ into the upper joint space in the treatment of temporomandibular joint (TMJ) internal derangements.

Design Randomised non-blinded prospective trial.

Intervention Thirty-one people (five males and 26 females, age 1453 years) who had TMJ problems in 41 joints were randomly divided into two treatment groups. One group received arthrocentesis alone, the other arthrocentesis plus intra-articular injection of SH. Both groups contained patients with disc displacement with reduction and with closed lock. Patients were evaluated before the procedure, on postoperative day 1 and then regularly for 24 months.

Outcome measures Intensity of TMJ pain, jaw function and clicking sounds in the TMJ were assessed using visual analogue scales (VAS). Maximal mouth opening and lateral jaw movements also were recorded at each follow-up visit.
Results Both techniques increased maximal mouth opening, lateral movements and function while reducing TMJ pain and noise.

Maximal jaw opening increased at 24 months in both groups (see Table 1).

Conclusions Patients benefited from both techniques but arthrocentesis with injection of $\mathrm{SH}$ seemed to be superior to arthrocentesis alone. Jaw function improved and maximal mouth opening increased earlier and continued longer in arthrocentesis and SH group.

Evidence-Based Dentistry (2002) 3, 70-71. doi:10.1038/ sj.ebd.6400123

Address for reprints: Dr Alpaslan, Ziyabey Caddesi, Dostlar Sitesi, C-Blok No. 112, Balgat, 06520 Ankara, Turkey. E-mail: alpaslans@tr.net

Table 1 Maximal jaw opening after treatment in subjects with TMJ problems

\begin{tabular}{|c|c|c|c|c|}
\hline \multirow[b]{2}{*}{ Time of visit } & \multicolumn{2}{|c|}{ Mean maximal opening in internal derangement patients* } & \multicolumn{2}{|c|}{ Mean maximal opening in closed lock patients* } \\
\hline & Arthrocentesis and $\mathrm{SH}$ & Arthrocentesis only & Arthrocentesis and $\mathrm{SH}$ & Arthrocentesis only \\
\hline Pre-operative & 34.3 & 31.1 & 28.4 & 24.0 \\
\hline Post-operative & 41.6 & 36.9 & 38.0 & 31.0 \\
\hline 24 months & 42.4 & 36.6 & 39.1 & 31.1 \\
\hline
\end{tabular}

* Standard deviation available in original paper.

$P<0.05$ within and between group analysis at post-operative and 24-month visits.

$\mathrm{SH}$, Sodium hyaluronate.

\section{Commentary}

This paper purports to show that arthrocentesis increases joint function and reduces pain in TMJ internal derangement and that the improvement is increased with the introduction of $\mathrm{SH}$, particularly in the closed lock case. It represents a carefully planned investigation. Figures for jaw function (opening and lateral excursion) are presented in tables for each of the preoperative and monthly examinations, but VAS for click and pain are presented in graph form. As the two test groups were each subdivided into subjects who had reduction and those who did not, the numbers in each group are small (19 and 22 joints in 16 and 15 patients. The months of suffering vary from 1-96 months, with the mean at 23.15 months.

Surprisingly, there was no discussion regarding the myogenous factors normally noted in TMJ pain patients which implies that the patients had only joint problems, a very unusual presentation. The diagnosis of internal 
derangement and closed lock was made on clinical grounds only and it is not clear whether patients with two joints affected had the same diagnosis for each joint or indeed had the same treatment for each joint. The only estimate of click was on a VAS and found to be 4 in a scale of 10 in the closed lock case, which reduced in the arthrocentesis-plus-SH treatment group and stayed unchanged in the arthrocentesis-alone group. There was no detailed description of how any of the measurements were made. It must be asked whether the closed lock was the correct and only diagnosis, for closed lock suggests that there should be no click before treatment. The investigators were not blinded to the method and there is no account of what other treatment or management was running concurrently. For instance, were subjects taking nonsteroidal anti-inflammatory drugs and were they encouraged to do TMJ exercises?

The paper does not make dramatic claims and both methods appear to give notable improvement in opening and pain. Those patients who received SH seemed to have the greater improvement, particularly those with the least opening initially. Nevertheless, the numbers in the groups are very small, the joints assessed were not always in different patients, and the initial diagnosis was presumptive only. This paper has too many weaknesses to arrive at reliable conclusions.

\section{Richard Juniper Department of Postgraduate Medical \& Dental Education, Oxford, UK}

